\title{
Arithmetical datatypes with true fractions
}

\author{
Jan A. Bergstra ${ }^{1} \cdot$ Alban Ponse $^{1}$
}

Received: 1 April 2019 / Accepted: 14 November 2019 / Published online: 13 January 2020

(C) The Author(s) 2020

\begin{abstract}
We consider several novel congruences on the signature of meadows with the aim to survey different notions of fractions. In particular we suggest a notion of "true fraction".
\end{abstract}

\section{Contents}

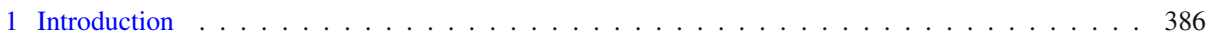

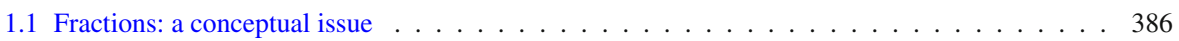

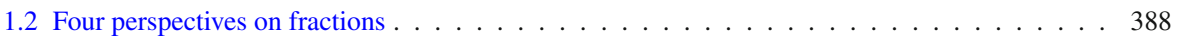

1.3 Related work on arithmetical datatypes . . . . . . . . . . . . . . . . . . . 390

2 Fracterm terminology . . . . . . . . . . . . . . . . . . . . . . . 390

2.1 Fracterms and fracpairs $\ldots \ldots \ldots \ldots \ldots \ldots$

2.2 Congruence-dependent properties of fracterms . . . . . . . . . . . . . . . . . . 391

2.3 Fracterm-related congruence properties . . . . . . . . . . . . . . . . . . . . . . . . . 391

2.4 A transversal for FFT-arithmetical datatypes . . . . . . . . . . . . . . . . . . . . . . . . . . . . . . . . . . . . . . . . . . . .

3 Fracpair transparency: some limitations . . . . . . . . . . . . . . . . . . . . . . 393

3.1 The case of involutive meadows . . . . . . . . . . . . . . . . . . . . . . . . 394

3.2 Fracterm transparency over a commutative ring . . . . . . . . . . . . . . . . . . . 394

4 Arithmetical datatypes with simple fracterms . . . . . . . . . . . . . . . . . . . 395

4.1 GCD-based addition . . . . . . . . . . . . . . . . . . . . . . . . 395

4.2 A model with CFAR . . . . . . . . . . . . . . . . . . . . . . . . . . . . . . . . . . 397

4.3 An arithmetical datatype with a simplifying operator . . . . . . . . . . . . . . . . 398

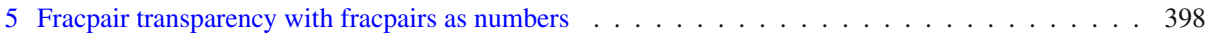

5.1 Two-dimensional vector meadows . . . . . . . . . . . . . . . . . . . . . . . . . . . . . 399

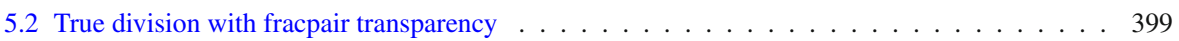

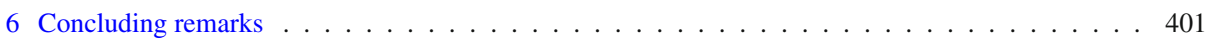

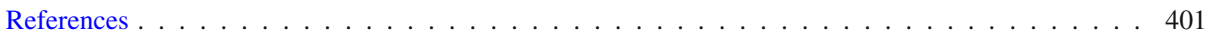

$凶$ Alban Ponse

a.ponse@uva.nl

Jan A. Bergstra

j.a.bergstra@uva.nl; janaldertb@gmail.com

1 Informatics Institute, University of Amsterdam, Amsterdam, The Netherlands 


\section{Introduction}

This paper is written in honour of Rob van Glabbeek on the occasion of his 60th birthday. We congratulate Rob with this milestone and with his outstanding performance in the area of process theory.

The idea of meadows is to introduce, on top of the signature of rings and fields, a function symbol for inverse, thus obtaining inversive notation $x^{-1}$, or for division, thereby obtaining divisive notation $\left(x / y\right.$, usually with the understanding that $\left.x^{-1}=1 / x\right)$, and to insist, or at least to prefer, that operations are total.

An arithmetical datatype, admittedly an informal notion, is an abstract datatype which provides sorts and functions closely related to arithmetic. ${ }^{1}$

Assuming that operations are total and that division is a named operation, the equational logic of known and sometimes novel structures is investigated. $\mathrm{Md}^{d}$, the equational axiomatisation of (commutative, regular and involutive) meadows with divisive notation, consists of the equations for a commutative ring CR (Table 1) plus three more axioms concerning division as displayed in Table 2.

Regularity ensures that a meadow which meets these three requirements is the expansion of a (von Neumann) regular ring with a division operation. Table 3 displays different levels of regularity.

For equational axioms for meadows see $[5,11,21,23]$ and for further theoretical information we refer to $[3,4] .^{2}$

\subsection{Fractions: a conceptual issue}

The question "what is a fraction?" is just as difficult as the question "what is a process?".

Process. In process algebra it is not uncommon to refer to $p \equiv a \| b$ as a concurrent process, with $a$ and $b$ atomic actions, and with $\equiv$ syntactic equality. Let $q \equiv a \cdot b+b \cdot a$. This equation gives rise to two issues. To begin with, considering $q$ a parallel process is unconvincing at first sight. Assuming arbitrary interleaving, however, one finds $p=q$. At this point the terminology becomes somewhat problematic: is it the case that $p$ is not a concurrent process after all, or conversely that $q$ is not a sequential process after all? The situation is clarified by referring to $p$ and $q$ as process expressions rather than as processes. Then under the assumption of arbitrary interleaving $p$ and $q$ denote the same process while in another semantic model (process algebra) they may not. A process algebra admits true concurrency if some process expressions involving parallel composition are not equivalent to any process expression made up of sequential features only.

A second issue arises because one may not be satisfied with the use of the terminology: one may prefer to refer to $p$ and $q$ as processes instead of as process expressions, and then to use phrases like, "the interpretation of process $p$ in model $M$ " in order to refer to specific elements of a process algebra.

1 Comparable to the terminology of process algebra, an arithmetical datatype might be called a number algebra, but we prefer not to do so because it seems to come as a default that algebras are about numbers.

2 Besides meadows as defined in [5] at least three other kinds of meadows can be distinguished: common meadows [9], wheels $[15,16,26]$, and transrationals [17]. The characteristic feature of meadows at large is the presence of either inverse or division as a function symbol in the signature, in addition to the signature of a ring. This difference with conventional approaches has significant impact on equational reasoning which it supports quite well, and it fits the ubiquitous use of division. When working with meadows, issues about division by zero and partiality can be studied with adequate precision. 
Table $1 \mathrm{CR}$, axioms for a commutative ring

$$
\begin{aligned}
& (x+y)+z=x+(y+z) \\
& x+y=y+x \\
& x+0=x \\
& x+(-x)=0 \\
& (x \cdot y) \cdot z=x \cdot(y \cdot z) \\
& x \cdot y=y \cdot x \\
& 1 \cdot x=x \\
& x \cdot(y+z)=x \cdot y+x \cdot z
\end{aligned}
$$

\begin{tabular}{|c|c|}
\hline $1 /(1 / x))=x$ & Involution \\
\hline$(x \cdot x) / x=x$ & Regularity \\
\hline$x / y=x \cdot(1 / y)$ & Factorisation of division \\
\hline$(x / x) \cdot(x / x)=x / x$ & Weak regularity \\
\hline$(x \cdot x) / x=x$ & Regularity \\
\hline$x /(x \cdot x)=1 / x$ & Coregularity \\
\hline$x \neq 0 \rightarrow x / x=1$ & Strong regularity \\
\hline
\end{tabular}

Table $2 \mathrm{Md}^{d}$, axioms for a divisive meadow

Table 3 Levels of regularity in divisive notation

Investigating the formidable richness of semantic models for even a very simple process notation has been a remarkable achievement of Rob van Glabbeek [27]. In that spirit, but by no means as comprehensive, we will look into the rich world of models of the syntax of meadows.

Fraction. Now for fractions: let $p \equiv 2 / 3$ and $q \equiv 4 / 6$. It is customary to consider $p$ to be a simplified fraction while $q$ is not a simplified fraction. Now in the meadow $\mathbb{Q}_{0}^{d}$ (rational numbers with divisive notation, and with zero-totalised division), one finds $p=q$. What can be concluded? Is $p$ to be considered not simplified after all, or has $q$ turned out to be simplified in hindsight, or something else?

The situation can be clarified by speaking of fraction expressions. Then $p$ and $q$ are different fraction expressions which denote the same value in $\mathbb{Q}_{0}^{d}$. Two such fraction expressions are called equivalent, taking characteristic zero for granted. A structure for the same signature that distinguishes $p$ and $q$ or any other pair of equivalent but non-identical fractions may be said to admit true fractions. Little is known about such structures, and below we will obtain some preliminary results.

The second issue mentioned above regarding processes has a counterpart in the case of fractions. One may prefer to understand fractions as expressions rather then primarily as elements of a datatype, and to refer to the interpretations of fractions in an arithmetical datatype as numbers.

It seems to be the case that in ordinary mathematics a fraction is considered an element of an algebra (for instance the field of fractions over an integral domain). Such elements, which may be called numbers or numbers of some specific kind, are not equipped with a numerator and a denominator. Merely representatives of these numbers (with numbers viewed as equivalence classes of representatives) are decomposable as expressions. This understanding of fractions explains why mathematical textbooks usually don't provide a 
definition of fractions. We refer to Fandiño Pinilla [18] for the viewpoint that fraction is not a mathematical concept in need of a precise definition.

Alternatively one may insist that a fraction comes with a nominator, a denominator, and a function symbol. This perspective, taking fractions for structured abstract entities, seems to be leading in the context of school arithmetic. The idea that fractions have structure creates a ramification of options, four of which will be mentioned. The least abstract perspective will be referred to as "fractions as terms".

\subsection{Four perspectives on fractions}

Fractions as values $(\mathrm{FaV})$ and fractions as terms $(\mathrm{FaT})$ represent two opposite perspectives on fractions which are both perfectly consistent, but which can hardly be reconciled, however. We prefer viewing fractions as terms. Two intermediate positions merit mention: to consider fractions as pairs of values (FaPV, taking the pair of the respective values of the terms meant in perspective $\mathrm{FaT}$ ), for instance rational numbers, and alternatively (FaPW) is to consider fractions as pairs of non-fractions, that is pairs of whole numbers or expressions for whole numbers, with the second element required to be non-zero or positive.

Fractions as values is the more abstract perspective of these, fractions as pairs of (possibly fractional) values is more abstract than fractions as terms. The relation between perspectives FaT, FaPV, and FaPW is not easily explained by way of levels of abstraction. One might say that both FaPV and FaPW correspond to instances of FaT.

We favour the fractions as terms perspective because it optimally supports theoretical work on fractions. The FaT perspective can be summarised as follows:

1. Each fraction comes with a unique nominator and a unique denominator as well as a unique leading function symbol. Nominator and denominator may be but need not be wholes.

2. An arithmetical datatype admits true fractions if it distinguishes some fractions which are equivalent (have the same value in a certain preferred arithmetical datatype such as the meadow of rational numbers).

3. The standard interpretation of fractions does not admit true fractions.

4. Fractions are not numbers in the conventional sense, that is, not elements of well-known rings and fields. Fractions rather exist in free term algebras.

5. For fractions $p$ and $q, p=q$ denotes fraction equivalence rather than fraction equality.

6. It is also common to say that fractions $p$ and $q$ are equal if $p=q$; this 'imprecision' is acceptable as long as problematic confusion is prevented.

7. If reasoning about the ontology of fractions happens to be important, this potential source of confusion should be proactively removed (an unusual matter both in school arithmetic and in academic arithmetic).

Of the intermediate perspectives mentioned above the FaPV perspective seems not to merit further attention, while FaPW has a significant audience in the educational world and if only for that reason it is of independent importance. When viewing fractions as pairs of values of wholes the incentive to view fractions as numbers or as a kind of numbers becomes stronger. We summarise the perspective of fractions as pairs of wholes as follows:

1. When viewing fractions as pairs of values of wholes, it is less plausible to view fractions as numbers.

2. Fractions are "number-like" in the following sense: fractions may be interpreted in an arithmetical datatype $\left(\widehat{\mathbb{Q}}_{0, g c d}^{d}\right.$ as introduced below) which is sufficiently similar to wellknown arithmetical datatypes to justify speaking of its elements as "a kind of number". 
3. Fractions may, if one so wishes, be considered "a kind of numbers" (though not "numbers", and also not "elements of a subsort of a sort of numbers"). This is an intermediate position between FaT and FaV, with a bias to FaPW.

Fracterms. A disadvantage of taking fractions for expressions is the connotation of formality and formal syntax which comes with such terminology. Instead of formal grammars, informative examples may be used as an inductive means for defining the concept of term, inductive in the sense of inductive logic rather than in the sense of a mathematical inductive definition. Different persons need not completely agree on what is a term or expression and on when two terms are considered the same.

In the sequel of the paper we will use the word fracterm to denote a term which has division as its leading function symbol. By using fracterm instead of fraction we avoid a commitment to a specific perspective on fractions.

Terms and notations; characteristic zero. For closed terms $t$ and $r$, syntactic equality takes all detail into account. A higher level of abstraction is useful for work on fractions. Equivalence of terms, while taking various practical notational conventions into account, written $t \equiv r$, provides some level of abstraction. By taking into account operator precedence, associativity of addition and multiplication, and removal of redundant bracket pairs, further abstraction is obtained. Although in this paper we will always write $t / r$ when considering fracterms, it is common to separate $t$ and $r$ with a horizontal bar, in which case versions of the division operator with lower precedence can be used.

It is understood that negation takes priority over addition and that both multiplication and division take priority over summation. Moreover addition and multiplication are supposed to associate to the left. Thus e.g. $1+1+1 \equiv(1+1)+1,1+(1+1) \cdot(1+1) \equiv 1+((1+1) \cdot(1+1))$ and $1+-1 \equiv 1+(-1)$. We will freely use integers in decimal notation as shorthands for the corresponding closed expressions, so that $2 \equiv 1+1,-2=-(1+1), 3 \equiv 1+1+1$ etc. If we have to be more specific, numerals $\underline{n}$ are used: $\underline{0}=0, \underline{1}=1$, and $\underline{n+1}=\underline{n}+1$. Expressions $n+m$ and $n \cdot m$ stand for the associated numeral.

Many models of $\mathrm{Md}^{d}$ exist. We will consider $\mathbb{Q}_{0}^{d}$, the meadow of rational numbers, which results from the field of rational numbers by expanding it with division made total by having $1 / 0=0$. As other useful structures, which however are not used in this paper, we mention (i) $\mathbb{Q}_{a}^{d}$, which results from the same field by making division total with $1 / 0=a$ for a new arithmetical entity $a$ which serves as an error element, $(i i) \mathbb{Q}_{\star}^{d}$ for the expansion of $\mathbb{Q}$ to a structure with a partial division function, $($ ii i $)$ for a prime number $p$, the finite meadows $\mathbb{F}_{p, 0}^{d}$ that result from expanding the finite field of characteristic $p$ with a division operator that is made total by setting $1 / 0=0$, and (iv) the corresponding structures $\mathbb{F}_{p, a}^{d}$, and $\mathbb{F}_{p, \star}^{d}$. These structures give rise to the following equivalences on fracterms: $={ }_{0}$ for $\mathbb{Q}_{0}^{d},={ }_{a}$ for $\mathbb{Q}_{a}^{d}$ and $=_{\star}$ for $\mathbb{Q}_{\star}^{d}$ as well as to $=_{p, 0},={ }_{p, a}$, and $=_{p, \star}$, in the case of finite characteristic $p$.

In mathematical and educational practice it is common to write $t=r$ for $=_{\star}$, although without the underlying idea that expressions over $\Sigma_{\mathrm{Md}^{d}}$ are involved, and without any commitment to a specific logic of partial functions.

In the present paper $1 / 0=0$ is made as a simplifying assumption, not as a matter of principle, nor as an expression of an insight in the nature of numbers and or division; it just helps to obtain equational specifications and axiom systems. The idea that division, or 
inverse, deserves its own function symbol is a matter of principle, however. ${ }^{3}$ An algebra for the signature $\Sigma_{\mathrm{Md}^{d}}$ or for some larger signature is also referred to as an arithmetical data type.

In order to simplify the discussion it is assumed that all arithmetical datatypes considered below have characteristic 0. Adapting the terminology as developed below to a finite characteristic $k$ is easy and most questions can be discussed for finite characteristic $k$ as well. Moreover, it is assumed that the integers have an ordering, which is not included in the signature of meadows, however. We make no use of the sign function which is introduced in [3] for that purpose.

\subsection{Related work on arithmetical datatypes}

Arithmetical datatypes may have different applications. The work in this paper contributes to the discussion concerning the question "what is a fraction?". This seemingly trivial question has led to many different answers and viewpoints in the educational literature, ranging from the proposal to forget about fractions in [28] to the influential classification of aspects of fractions in [20]. As stated before (Sect. 1.2), at least four paradigms on fractions can be distinguished: fractions as values, fractions as pairs of whole numbers [22], ${ }^{4}$, and fractions as terms. Viewing fractions as values is advocated in [25]. In [1] an excursion is made where a logic of fractions is worked out as a paraconsistent logic. In [8] it is investigated in which meadows the collection of fractional elements is closed under addition. In [2] it is shown that fractions involving a single variable can be brought in so-called mixed fraction format. The paper [10] is devoted to one particular option for the fractions-as-pairs paradigm. Our work on arithmetical datatypes started with an initial algebra specification of the abstract datatype of rational numbers as presented in [11].

\section{Fracterm terminology}

In this section we survey terminology on fracterms.

\subsection{Fracterms and fracpairs}

In $[1,2,8]$ a fraction is understood as a fracterm (a term with division as a leading function symbol).

\footnotetext{
3 The relevance of having division or inverse in the signature can be seen as follows for instance: the class of models of $\mathrm{Md}^{d}$ is an elementary class, while the class of its reducts to the signature of rings, that is, the class of regular rings is not an elementary class.

4 In Lortie-Forgues et al. [22] it is noticed that:

A fraction has three parts, a numerator, a denominator, and a line separating the two numbers. This configuration makes fraction notation somewhat difficult to understand. For instance, students, especially in the early stages of learning, often misread fractions as two distinct whole numbers (e.g., 1/2 as 1 and 2), as a familiar arithmetic operation (e.g., $1+2$ ) or as a single number (e.g., 12)......

According to these authors not only is a fraction not a single number, it is not (like the result of) a familiar operation on numbers either, and it is not a pair. These remarks provide ample evidence of the difficulty of defining a fraction in unambiguously positive terms.
} 
Fracterms exist in a context of other related notions and terminology, which are introduced stepwise below. ${ }^{5}$

Definition 2.1.1 A term over the signature of meadows is a wholeterm if it contains no occurrence of the division function.

A wholeterm $t$ is simple if it is 0 or either has the form $r$ or the form $-(r)$ with $r$ made up from the constant 1 and addition only. In the first case $t$ is a positive wholeterm, in the second case $t$ is a negative wholeterm.

Definition 2.1.2 A term over the signature of meadows is a fracterm if its leading function symbol is division. The arguments of the leading function symbol are termed numerator and denominator, respectively.

Definition 2.1.3 (Flat fracterm) A fracterm is flat if its numerator and denominator are wholeterms.

Definition 2.1.4 (Simple fracterm) A fracterm is simple if it is a flat fracterm of which the numerator is a simple wholeterm and the denominator is a positive simple wholeterm.

Definition 2.1.5 A fracpair is a simple fracterm.

Remark: the notion of a fracpair depends on the context. In [10] a more liberal definition of fracpairs is used, allowing the denominator to be 0 or negative.

\subsection{Congruence-dependent properties of fracterms}

Some properties of fracterms (or of relevance to fracterms) are dependent on a given congruence. We write $\cong$ for an arbitrary congruence on closed meadow terms. Among these properties are the following:

Definition 2.2.1 The characteristic of $\cong$ is the smallest positive integer $n$ such that $\underline{n} / 1 \cong$ $0 / 1$ if it exists and 0 otherwise.

As was stated in Sect. 1.2 above, the discussion below will be limited to the case of characteristic 0 .

Definition 2.2.2 (Division safety) A closed expression $t$ is division unsafe for a congruence $\cong$ if it has a subterm $r / s$ such that $s \cong 0$. A closed term is division safe w.r.t. $\cong$ if it is not division unsafe.

Definition 2.2.3 (Simplified fracpair) A fracpair $\underline{n} / \underline{m}$ or $(-\underline{n}) / \underline{m}$ is simplified if $\operatorname{gcd}(n, m)=$ 1.

\subsection{Fracterm-related congruence properties}

Several useful properties of congruences relate to fracterms. We will write $\mathbb{Q}_{\cong}^{d}$ for $T_{\Sigma_{\mathrm{Md}^{d}}} \cong$, the set of closed terms over $\Sigma_{\mathrm{Md}^{d}}$ modulo $\cong$.

5 Moving from the language of fracterms and fracpairs to fractions (thereby adopting a fractions as terms perspective) is easy: the idea is that by substituting fraction for fracterm, and also substituting fraction for fracpair a workable terminology of fractions is obtained. 
Flattening (to fracpair form) A congruence $\cong$ admits flattening to fracpair form if for each closed term $t$ there is a fracpair $s$ with $t \cong s$.

Completeness for wholeterms A congruence $\cong$ is complete for wholeterms if for all division-free closed expressions $t$ and $r, t \cong r \Longleftrightarrow \mathbb{Z} \models t=r$.

Value protection A congruence $\cong$ is value protecting if for all closed terms $t$ and $r$, if $t \cong r$, then $\mathbb{Q}_{0}^{d} \models t=r$.

(In other words: $\mathbb{Q}_{0}^{d}$ is a homomorphic image of $\mathbb{Q}_{\cong}^{d}$ ).

Componentwise multiplication for fracterms A congruence $\cong$ allows componentwise multiplication for fracterms if

$$
\mathbb{Q}^{\stackrel{d}{=}} \models(x / y) \cdot(u / v)=(x \cdot u) /(y \cdot v) .
$$

Conventional negation A congruence $\cong$ conforms to conventional negation if $\mathbb{Q}_{\cong}^{d} \models$ $-(x / y)=(-x) / y$.

Uniform division by zero $\mathrm{A} \cong$ enjoys uniform division by zero if for all $t, t / 0 \cong t \cdot(1 / 0){ }^{6}$ Fracpair separation A congruence $\cong$ separates fracpairs $p$ and $q$ if $\mathbb{Q}_{0}^{d} \models p=q$ and $p \nsucceq q$. A typical example is the separation of $0 / 1$ and $0 / 2$.

Fracpair transparency A congruence $\cong$ is transparent for fracpairs if for all fracpairs $t / r$ and $s / u, t / r \cong s / u$ implies $t \cong s$ and $r \cong u$.

Full fracterm transparency A congruence $\cong$ is transparent for fracterms if for all closed and division safe fracterms $t / r$ and $s / u$ it is the case that $t / r \cong s / u$ implies $t \cong s$ and $r \cong u$.

$\mathbb{Q}_{0}^{d}$ is not transparent for fracpairs, rather the opposite: no pair of simple fractions with the same value in $\mathbb{Q}_{0}^{d}$ is distinguished in $\mathbb{Q}_{0}^{d}$. The initial algebra of $\mathbf{M d}^{d}$, however, distinguishes $\underline{2} / \underline{2}$ and $1 / 1$, and therefore features some limited form of transparency for fracpairs (limited because $\underline{4} / \underline{2}=\underline{2} / 1$ ). We refer to [13] for relevant information in said initial algebra.

Full fracterm transparency, beyond fracpair transparency, excludes flattening. Otherwise e.g. flattening yields that $(1 / \underline{2}) /(1 / \underline{2}) \cong \underline{n} / \underline{n}$ for some $n \in \mathbb{N}^{+}$. Full fracterm transparency yields $1 / \underline{2} \cong \underline{n}$ which cannot be true in $\mathbb{Q}_{0}^{d}$.

True division A division operation is a true division operation if it separates some fracterms $p$ and $q$ while $\mathbb{Q}_{0}^{d} \models p=q$. In this case, $p$ and $q$ are true fractions.

Zero-totalised division Division is zero-totalised if $t / 0 \cong 0$ for all $t$.

Quasi cardinality rule (QCR). The congruence satisfies the quasi cardinality rule (QCR) if $\mathbb{Q}^{d} \models x / y+z / y=(x+z) / y .^{7}$

A restricted version $\mathrm{QCR}^{0}$ of QCR reads: $0 / y+0 / y=0 / y$, while a stronger version of $\mathrm{QCR}, \mathrm{QCR}^{+}$reads: $v \neq 0 \rightarrow x / y+z /(v \cdot y)=((v \cdot x)+z) /(v \cdot y)$.

Conditional fracterm addition rule (CFAR) The conditional fracterm addition rule (CFAR) expresses the most well-known rule for addition:

$$
\mathbb{Q}^{d} \cong y \neq 0 \& u \neq 0 \rightarrow x / y+z / u=(x \cdot u+y \cdot z) /(y \cdot u)
$$

CFAR is not compatible with QCR or its variants in a setting with full fracterm separation.

\footnotetext{
6 Division by zero is uniform in the involutive meadows of [5], as well as the non-involutive regular meadows of [7] and in the common meadows (which are noninvolutive and coregular) of [9]. In these cases, however, also the stronger requirement that $t / r \cong t \cdot(1 / r)$ is satisfied. The latter requirement, however, will be relaxed below in order to deal with true fractions.

7 QCR is a phrase ascribed to Griesel [19] in Padberg [24]. QCR is considered a most illuminating feature of the concept of fractions. QCR formalises the popular explanation of fractions by means of pies and cakes.
} 
Flattening to fracpair form is not implied by any combinations of equations which are true in all meadows that are expansions of a field with a 0-totalised division operator. If such equations $E$ existed, then these would be derivable from $\mathrm{Md}^{d}$ given the completeness theorem for $\mathrm{Md}$ (see [5,6] for an adaptation to divisive notation), which then implies that $\mathrm{Md}^{d} \vdash E$. It then follows that all minimal models, including the initial algebra of $\mathrm{Md}^{d}$ admit flattening. The latter is not the case according to [13].

\section{Proposition 2.3.1 Relative to $\mathrm{Md}^{d}$, CFAR is equivalent with strong regularity.}

Proof Strong regularity implies CFAR: if $y \neq 0, u \neq 0$ then $x / y+z / u=x / y \cdot u / u+z / u$. $y / y=(x \cdot u+y \cdot z) /(y \cdot u)$. Conversely let $x \neq 0$. We may assume $1 \neq 0$, then, with $y=x$, $u=z=1$ we find $x / x+1 / 1=(x \cdot 1+x \cdot 1) /(x \cdot 1)=(x+x) / x$ and subtracting $x / x$ from both sides yields $1=1 / 1=x / x$.

\subsection{A transversal for FFT-arithmetical datatypes}

We will focus on arithmetical datatypes with signature $\Sigma_{\mathrm{Md}^{d}}$, in which the integers (with 0 and addition) constitute a commutative ring.

Under the assumptions of minimality (no proper substructures), flattening and fracpair transparency, the following representation theorem is useful. We refer to the class of such datatypes as FFT-arithmetical datatypes. In the terminology of abstract datatypes a transversal is a family of expressions which provides a unique notation for each element of a datatype. The fracpairs constitute a transversal for an FFT-arithmetical datatype. As stated in Sect. 1.2 (in paragraph Terms and notations; characteristic zero), we assume a fixed choice of unique notations for integers (e.g. $-\underline{3} \equiv-((1+1)+1)$ and so on). The following works for arbitrary characteristic.

Proposition 2.4.1 In an FFT-arithmetical datatype $\mathbb{A}$ : (i) different fracpairs have a different interpretation, and (ii) each element is the interpretation of some fracpair.

Proof If $p / q$ and $r / s$ are fracpairs then with fracpair transparency $\mathbb{A} \models p=q$ implies $p \equiv r$ and $q \equiv s$. Thus no pair of different fracpairs is identified in $\mathbb{A}$.

Moreover flattening implies that each closed term is equal in $\mathbb{A}$ to some fracpair. Minimality implies that all elements of the domain for $\mathbb{A}$ are equal to a closed term.

Clearly all datatypes with a commutative ring of integers and meeting the two criteria of Proposition 2.4.1 are FFT-arithmetical datatypes.

Below we will focus on FFT-arithmetical datatypes with characteristic 0 (without mention of the prefix FFT). When designing an FFT-arithmetical datatype the various ring operations are known on all integers and the division operation is known (predetermined) on all pairs of arguments $n$ (the interpretation of $\underline{n}$ ) and $m$ with $n \in \mathbb{Z}, m \in \mathbb{N}^{+}$, so that a fracpair $\underline{n} / \underline{m}$ (with $n \in \mathbb{Z}, m \in \mathbb{N}^{+}$) denotes the rational number $n / m$. In principle all other values of the operations are free, that is, only constrained by axioms one wishes to adopt.

\section{Fracpair transparency: some limitations}

We first consider the impossibility of obtaining fracpair transparency in an involutive meadow, and then the weaker case of fracterms over a commutative ring. 


\subsection{The case of involutive meadows}

We will insist that the admissibility of flattening is an essential property for a calculus of fracterms. Now the initial meadow does not admit flattening to fracpair form (see e.g. [13]). Moreover, no homomorphic image of the initial meadow except $\mathbb{Q}_{0}^{d}$ provides flattening.

Proposition 3.1.1 Let $\mathbb{M}_{0}$ be a minimal involutive meadow (i.e. a model of $\mathrm{Md}^{d}$ ). If the congruence of $\mathbb{M}_{0}$ admits flattening, then $\mathbb{M}_{0}$ satisfies strong regularity (and conversely).

Proof Let $p$ be a prime number differing from the characteristic $c$ of $\mathbb{M}_{0}$. As $\mathbb{M}_{0}$ admits flattening there must be non-zero natural numbers $n$ and $m$ such that $1-p / p=\underline{n} / \underline{m}$.

Due to value protection it must be the case that in characteristic $c, \mathbb{F}_{c, 0}^{d} \models \underline{n} / \underline{m}=0$ and also (by multiplying both sides with $\underline{m^{2}}$ ), $\mathbb{F}_{c, 0}^{d} \models \underline{n} \cdot \underline{m}=0$, i.e. $n \cdot m=0 \bmod c$ so that in $\mathbb{M}_{0}, \underline{n} / \underline{m}=((\underline{n} \cdot \underline{n}) / \underline{n}) / \underline{m}=(\underline{n} \cdot \underline{n}) /(\underline{n} \cdot \underline{m})=(\underline{n} \cdot \underline{n}) /(\underline{n} \cdot m)=0$. It follows that $\mathbb{M}_{0} \models 1-p / p=0$ and thus (using the equations of $\left.\overline{M^{2}} d^{d}\right) \mathbb{M}_{0} \models p / p=1$. From this it follows that all fracterms can be simplified in the congruence of $\mathbb{M}_{0}$, which in turn implies that $\mathbb{M}_{0}$ is strongly regular (is a cancellation meadow in the terminology of [3]).

It follows from the above proposition that in an arithmetical datatype which satisfies $\mathrm{Md}^{d}$ and admits flattening, no instance of fracpair separation can be expected. In the remainder of the paper we will consider fracpair transparency in a larger class of arithmetical datatypes.

\subsection{Fracterm transparency over a commutative ring}

Several slightly stronger negative results can be formulated, upon relaxing the requirement that one works in a meadow.

Proposition 3.2.1 Let $\mathbb{M}$ be an arithmetical algebra and a proper homomorphic preimage of $\mathbb{Q}_{0}^{d}$. Suppose that $\cong_{\mathbb{M}}$ is flattening. Then:

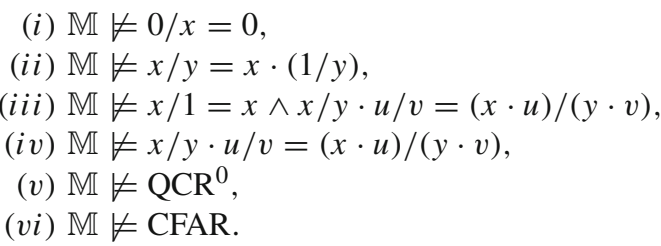

Proof (i) Assume that $\mathbb{M} \models \underline{k} / \underline{l}=\underline{n} / \underline{m}$ with $k, l, m, n \in \mathbb{Z}$ and $l, m>0$. One finds $\underline{k} / \underline{l}-\underline{n} / \underline{m}=\underline{a} / \underline{b}$ with $b>0$. Value protection yields that $a=0$. With $0 / x=0$ this yields $\underline{k} / \underline{l}-\underline{n} / \underline{m}=0$. This implies that $\mathbb{M} \cong \mathbb{Q}_{0}^{d}$, and thus contradicts the assumption that $\mathbb{M}$ is a proper homomorphic preimage of $\mathbb{Q}_{0}^{d}$.

(ii) From $x \cdot(1 / y)=x / y$ one obtains $0 \cdot(1 / y)=0 / y$, and with CR that yields $0=0 / y$ thereby reducing this case to case $(i)$.

(iii) Reduction to case $($ ii $): x \cdot(1 / y)=(x / 1) \cdot(1 / y)=(x \cdot 1) /(1 \cdot y)=x / y$.

(iv) An improvement of case (iii): with flattening and fracpair transparency find $k \in \mathbb{N}^{+}$ such that $(0 / \underline{2})+(1 / \underline{3})=\underline{k} / \underline{3} \cdot k$. Then

$$
0 / \underline{2}=(\underline{k} / \underline{3} \cdot k)-(1 / \underline{3})=(\underline{k} / \underline{k}) \cdot(1 / 3)-(1 / 1) \cdot(1 / 3)=((\underline{k} / \underline{k})-(1 / 1)) \cdot(1 / 3) .
$$

Now find $l \in \mathbb{N}^{+}$such that $(\underline{k} / \underline{k})-(1 / 1)=0 / \underline{l}$. Then $((\underline{k} / \underline{k})-(1 / 1)) \cdot(1 / 3)=(0 / \underline{l}) \cdot(1 / 3)=$ $0 /(\underline{l \cdot 3})$. Fracpair transparency implies $\underline{2}=\underline{l \cdot 3}$ which is impossible for a natural number $l$. 
(v) With $\mathrm{QCR}^{0}$ and $\mathrm{CR}, 0 / x+0 / x=(0+0) / x=0 / x$, so that with $\mathrm{CR} 0 / x=0$ whereby the case is reduced to case $(i)$.

(vi) With flattening find $z \in \mathbb{N}^{+}$such that $0=0 / \underline{z}$. Now $0 / \underline{z}=0=0+0=(0 / \underline{z})+$ $(0 / \underline{z})=0 / z^{2}$. Fracpair transparency gives $z=1$ (as $z>0$ ), whence $0=0 / 1$.

Flattening with value protection implies the existence of a $k>0$ such that $-(0 / 2)=0 / \underline{k}$. Now $0 / 1=0=(0 / \underline{2})+(-(0 / \underline{2}))=(0 / \underline{2})+(0 / \underline{k})=0 / \underline{2} \cdot k$, thus contradicting fracpair transparency.

The following questions remain unanswered, however.

Problem 3.2.2 Is there a homomorphic preimage of $\mathbb{Q}_{0}^{d}$ which (i) satisfies $\mathrm{CR}$, (ii) admits flattening, and (iii) contains at least one true fraction?

Problem 3.2.3 Is there a homomorphic preimage of $\mathbb{Q}_{0}^{d}$ which (i) satisfies $\mathrm{CR},($ ii $)$ admits flattening, and (iii) is transparent for fracpairs?

\section{Arithmetical datatypes with simple fracterms}

We are able to obtain positive results concerning the existence of arithmetical datatypes of characteristic 0 with flattening and fracpair transparency by compromising the axioms of CR. We will provide three examples.

\subsection{GCD-based addition}

The arithmetical datatype $\widehat{\mathbb{Q}}_{0, \text { gcd }}^{d}$ is given as follows:

- The domain $V$ is the same as for rational numbers but without imposing an equivalence relation on it: $V=\mathbb{Z} \times \mathbb{N}^{+}$.

- Constants and operations are as follows:

$$
\llbracket 0 \rrbracket=(0,1) \text { and } \llbracket 1 \rrbracket=(1,1),
$$

and with $\llbracket p \rrbracket=(a, b), \llbracket q \rrbracket=(e, f)$ :

$$
\begin{array}{rlrl}
\llbracket-(p) \rrbracket & =(-a, b) & (=\hat{-}(\llbracket p \rrbracket)), \\
\llbracket p \cdot q \rrbracket & =(a \cdot e, b \cdot f) & (=\llbracket p \rrbracket \hat{\bullet} \llbracket q), \\
\llbracket p+q \rrbracket & =((a \cdot f+b \cdot e) \backslash g c d(b, f),(b \cdot f) \backslash g c d(b, f)) & (=\llbracket p \rrbracket \hat{+} \llbracket q \rrbracket), \\
\llbracket p / q \rrbracket & =\left\{\begin{array}{ll}
(a \cdot f, b \cdot e) & \text { if } e>0 \\
(-(a \cdot f),-(b \cdot e)) & \text { if } e<0 \\
(0, b \cdot f) & \text { if } e=0
\end{array}(=\llbracket p \rrbracket \hat{/} \llbracket q \rrbracket),\right.
\end{array}
$$

where $\operatorname{gcd}(0, n)=n$ and $\backslash$ is integer division, thus $0 \backslash 3=0,6 \backslash 3=8 \backslash 4=2,-5 \backslash 5=$ -1 , etc, and because integer division should be a total operation in meadows, $-9 \backslash 2=$ $-5,7 \backslash 3=2$ and so on. For division the following alternative is plausible as well:

$$
(1,1) \hat{/}(0, b)=(0,1) \text { for all } b>0 .
$$

We prefer $(1,1) \hat{\jmath}(0, b)=(0, b)$ because that particular choice respects $1 /(1 / x)=x$ (i.e., the involution axiom in Table 2). 
Table $4 \widehat{C R}$ : axioms for a compromised commutative ring

$$
\begin{aligned}
& (x+y)+z=x+(y+z) \\
& x+y=y+x \\
& x+0=x \\
& -x=(-1) \cdot x \\
& 1+(-1)=0 \\
& (x \cdot y) \cdot z=x \cdot(y \cdot z) \\
& x \cdot y=y \cdot x \\
& 1 \cdot x=x \\
& x \cdot(y+z)=x \cdot y+x \cdot z
\end{aligned}
$$

The following properties are easy to demonstrate, where $\cong_{g c d}$ is the congruence induced by $\widehat{\mathbb{Q}}_{0, g c d}^{d}$ :

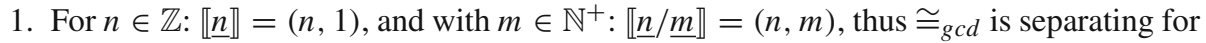
simple fracterms (i.e. fracpairs).

Indeed for $m, n \in \mathbb{Z}$, and $k, l \in \mathbb{N}$ : if $\widehat{\mathbb{Q}}_{0, g c d}^{d} \models \underline{m} / \underline{k}=\underline{n} / \underline{l}$, then $m=n$ and $k=l$.

2. The congruence $\cong_{g c d}$ is not fully fracterm transparent. Indeed consider $p \equiv(1 / \underline{2}) /(1 / \underline{2})$ and $q \equiv \underline{2} / \underline{2}$. Then $p \cong \cong_{g c d} q$, but not $1 / \underline{2} \cong g c d \underline{2}$.

3. The congruence $\cong_{g c d}$ admits flattening to fracpair form.

4. $\mathbb{Q}_{0}^{d}$ is a homomorphic image of $\widehat{\mathbb{Q}}_{0, \text { gcd }}^{d}$.

5. $\widehat{\mathbb{Q}}_{0, g c d}^{d}$ satisfies the equations of Table 4 (below, we show distributivity). From these equations the following equations can be derived: $0 \cdot 0=0,0 \cdot 1=0$, and $-0=0$.

6. $\widehat{\mathbb{Q}}_{0, \text { gcd }}^{d}$ satisfies the equations and open formulae of Table 5.

7. In $\widehat{\mathbb{Q}}_{0, g c d}^{d}$, multiplication is not componentwise for fracterms, division is not zero-totalised $((1 / \underline{2}) / 0=(1 / \underline{2}) \cdot 0=0 / \underline{2} \neq 0)$, and CFAR is not satisfied. Furthermore, $x=1 / \underline{2}$ refutes $x+(-x)=0$ and $0 \cdot x=0$.

8. $\widehat{\mathbb{Q}}_{0, g c d}^{d}$ satisfies characteristic 0 , completeness for wholeterms, value protection, conventional negation, uniform division by zero, fracpair transparency, QCR, and $\mathrm{QCR}^{+}$.

9. $\widehat{\mathbb{Q}}_{0, g c d}^{d}$ is not regular, for example $(\underline{2} \cdot \underline{2}) / \underline{2} \neq \underline{2}$.

Except distributivity, all equations in Table 4 are easily verified. For distributivity assume $\llbracket p \rrbracket=(a, b), \llbracket q \rrbracket=(e, f), \llbracket r \rrbracket=(u, v)$. Then

$$
\begin{aligned}
p & \cdot(q+r) \\
& =(a, b) \hat{\bullet}((e \cdot v+f \cdot u) \backslash g c d(f, v),(f \cdot v) \backslash g c d(f, v)) \\
& =((a \cdot e \cdot v+a \cdot f \cdot u) \backslash g c d(f, v),(b \cdot f \cdot v) \backslash g c d(f, v)) \\
& =((a \cdot e \cdot b \cdot v+b \cdot f \cdot a \cdot u) \backslash b \cdot g c d(f, v),(b \cdot f \cdot b \cdot v) \backslash b \cdot \operatorname{gcd}(f, v)) \\
& =((a \cdot e \cdot b \cdot v+b \cdot f \cdot a \cdot u) \backslash g c d(b \cdot f, b \cdot v),(b \cdot f \cdot b \cdot v) \backslash g c d(b \cdot f, b \cdot v)) \\
& =(a \cdot e, b \cdot f) \hat{+}(a \cdot u, b \cdot v) \\
& =(a, b) \hat{\cdot}(e, f) \hat{+}(a, b) \hat{)}(u, v) \\
& =p \cdot q+p \cdot r .
\end{aligned}
$$


Table 5 Some equations and formulae valid in $\widehat{\mathbb{Q}}_{0, \text { gcd }}^{d}$

\begin{tabular}{l}
$x / y=x \cdot(1 / y)$ \\
$1 /(1 / x)=x$ \\
$1 / 1=1$ \\
$x+(-x)=0 \cdot x$ \\
$x^{2}=x \cdot x$ \\
$0 \cdot\left(1+x^{2}+y^{2}+z^{2}+u^{2}\right) \neq 1+x^{2}+y^{2}+z^{2}+u^{2}$ \\
$0 \cdot x \neq x \rightarrow 0 \cdot(1 / x) \neq 1 / x$ \\
$1 /(0 / x)=0 \cdot(1 / x)$ \\
$0 \cdot x \neq x \wedge 0 \cdot y \neq y \rightarrow 0 \cdot x \cdot y \neq x \cdot y$ \\
$x \neq 0 \wedge y \neq 0 \rightarrow x \cdot y \neq 0$ \\
$0 \cdot x \cdot y \neq x \cdot y \rightarrow 1 /(x \cdot y)=(1 / x) \cdot(1 / y)$ \\
$0 \cdot x \cdot y \neq x \cdot y \rightarrow 1 /(x / y)=y / x$ \\
$0 \cdot y \cdot z \neq x \cdot y \rightarrow(x / y) / z=x /(y \cdot z)$ \\
\hline
\end{tabular}

\subsection{A model with CFAR}

The arithmetical datatype $\widehat{\mathbb{Q}}_{0, c f a r^{-}}^{d}$ is given as follows:

- The domain $V$ is the same as for rational numbers but without imposing an equivalence relation on it: $V=\mathbb{Z} \times \mathbb{N}^{+}$.

- Constants and operations are as follows:

$$
\llbracket 0 \rrbracket=(0,1) \text { and } \llbracket 1 \rrbracket=(1,1),
$$

and with $\llbracket p \rrbracket=(a, b), \llbracket q \rrbracket=(e, f)$ :

$$
\begin{array}{rlrl}
\llbracket-(p) \rrbracket & =(-a, b) & & (=\simeq(\llbracket p \rrbracket)), \\
\llbracket p \cdot q \rrbracket & =(a \cdot e, b \cdot f) & (=\llbracket p \rrbracket \cdot \llbracket q \rrbracket), \\
\llbracket p+q \rrbracket & =(a \cdot f+b \cdot e, b \cdot f) & & (=\llbracket p \rrbracket \tilde{+} \llbracket q \rrbracket), \\
\llbracket p / q \rrbracket & = \begin{cases}(a \cdot f, b \cdot e) & \text { if } e>0 \\
(-(a \cdot f),-(b \cdot e)) & \text { if } e<0 \\
(0, b \cdot f) & \text { if } e=0\end{cases}
\end{array}
$$

For division, the following alternative is plausible as well:

$$
(1,1) \tilde{f}(0, b)=(0,1) \text { for all } b>0 .
$$

As before, we prefer $(1,1) \tilde{/}(0, b)=(0, b)$ because that particular choice respects $1 /(1 / x)=x$.

A weak variant of CFAR is $\mathrm{CFAR}^{-}$:

$$
0 . y=0 \wedge 0 . u=0 \rightarrow x / y+z / u=(x \cdot y+y \cdot z) /(y \cdot u)
$$

Write $\cong_{c f a r^{-}}$for the congruence induced by $\widehat{\mathbb{Q}}_{0, c f a r^{-}}^{d}$. The congruence $\cong_{c f a r^{-}}$satisfies properties $1-4$ mentioned in the previous section. Further properties of $\cong_{c f a r}$ are the following: 
1. $\widehat{\mathbb{Q}}_{0, c f a r^{-}}^{d}$ satisfies the equations of Table 4 , except distributivity.

2. $\widehat{\mathbb{Q}}_{0, c f a r^{-}}^{d}$ satisfies the equations and open formulae of Table 5.

3. In $\widehat{\mathbb{Q}}_{0, c f a r^{-}}^{d}$, division is not zero-totalised $((1 / \underline{2}) / 0=(1 / \underline{2}) \cdot 0=0 / \underline{2} \neq 0)$, and QCR, $\mathrm{QCR}^{+}$are not satisfied.

4. $\widehat{\mathbb{Q}}_{0, c f a r^{-}}^{d}$ does not satisfy CFAR.

5. $\widehat{\mathbb{Q}}_{0, c f a r^{-}}^{d}$ satisfies characteristic 0 , completeness for wholeterms, value protection, componentwise multiplication, conventional negation, uniform division by zero, simple fracterm transparency, and $\mathrm{CFAR}^{-}$.

\subsection{An arithmetical datatype with a simplifying operator}

We briefly discuss another model with the same domain $V$ as in the previous two sections, but which uses a simplification operator. The operator sim simplifies pairs as follows (with $\left.n \in \mathbb{N}, m \in \mathbb{N}^{+}\right)$:

$$
\begin{aligned}
\operatorname{sim}(n, m) & =((n \backslash \operatorname{gcd}(n, m)) /(m \backslash \operatorname{gcd}(n, m))), \\
\operatorname{sim}(-n, m) & =(-(n \backslash \operatorname{gcd}(n, m)) /(m \backslash \operatorname{gcd}(n, m))) .
\end{aligned}
$$

For example, $\operatorname{gcd}(0,3)=3$ and thus $\operatorname{sim}(0,3)=(0,1)$.

Constants and operations can be defined as before, simplifying all results of operators except for division, which for that reason cannot be defined via inverse and multiplication. This yields a model in which many common laws are not valid $(1 \cdot x=x$, factorisation of division, $x+0=x$ ), although this model does satisfy $x+(-x)=0$. We refrain from discussing this model in detail.

\section{Fracpair transparency with fracpairs as numbers}

In this section we will outline another path towards modelling true fractions. Instead of considering weaker congruences for arithmetical expressions we consider a more liberal notion of number, effectively allowing to tag the elements of $\mathbb{Q}_{0}^{d}$ with additional information, which is informative about the choice of simple fraction one has in mind.

Thus assuming that the value of the fracpair $2 / 3$ is a rational number, the fact that it has numerator 2 and denominator 3 (rather than e.g. 4 and 6 respectively) may be added to it in some form of extension or expansion of the rational numbers which allows for the incorporation of additional information.

For an integer $n$ and a positive natural $m$ we will consider $n / f p m$ as a "kind of number", which is understood as a constant for an element of an extension of the meadow of rationals. The interpretation of $n / f p m$ must at the same time represent the value of $n / m$ and the values of $n$ and $m$.

Instead of a "fractions as terms" perspective this section focuses on the fractions as values perspective where values allow to encode additional information.

As it turns out there is much freedom in the choice of an extension for the meadow of rationals. We will discuss two cases: the first case (Sect. 5.1) allows separation of most fracpairs, and the second case (Sect. 5.2) allows separation of all fracpairs. 
Table 6 Complete basis of orthogonal idempotents

$$
\begin{aligned}
& v \cdot v=v \\
& v+d=1
\end{aligned}
$$

\subsection{Two-dimensional vector meadows}

We introduce two new constants, $v$ (value) and $d$ (decoration) for orthogonal idempotents in a (not strongly regular) meadow, both of which are assumed to be nonzero. Axioms for these constants are in Table 6 . Note that with CR these axioms imply $\mathrm{v} \cdot \mathrm{d}=0$ and $\mathrm{d} \cdot \mathrm{d}=\mathrm{d}$.

The direct sum $v \cdot \mathbb{Q}_{0}^{d} \oplus \mathrm{d} \cdot \mathbb{R}_{0}^{d}$ is an instance of what we call a vector meadow, and has elements $v \cdot p+\mathrm{d} \cdot q$ with $p \in \mathbb{Q}_{0}^{d}$ and $q \in \mathbb{R}_{0}^{d}$, and in which sum, product and division are defined componentwise, so

$$
\left(\mathrm{v} \cdot x+\mathrm{d} \cdot x^{\prime}\right) /\left(\mathrm{v} \cdot y+\mathrm{d} \cdot y^{\prime}\right)=\mathrm{v} \cdot(x / y)+\mathrm{d} \cdot\left(x^{\prime} / y^{\prime}\right) .
$$

The structure $\mathbf{v} \cdot \mathbb{Q}_{0}^{d} \oplus \mathrm{d} \cdot \mathbb{R}_{0}^{d}$ comes with two unary mappings: $\operatorname{val}(-)$ to $\mathbb{Q}_{0}^{d}$, named value part, and data $(-)$ to $\mathbb{R}_{0}^{d}$, named data part: $\operatorname{val}(\mathrm{v} \cdot p+\mathrm{d} \cdot q)=p$ and $\operatorname{data}(\mathrm{v} \cdot p+\mathrm{d} \cdot q)=q$. This structure is not strongly regular, as $d$ is non-zero and $d / d \neq 1$ (it easily follows that $d / d=d)$. However, this structure has useful strongly regular submeadows.

Proposition 5.1.1 Let $\alpha_{1}, \ldots, \alpha_{n}$ be a set of (positive) transcendent real number which are algebraically independent, and $q_{1}, \ldots, q_{n}$ a sequence of rational numbers.

The minimal submeadow $\mathbb{H}$ of $\mathrm{v} \cdot \mathbb{Q}_{0}^{d} \oplus d \cdot \mathbb{R}_{0}^{d}$ generated by $\beta_{i}(i \in\{1, \ldots, n\})$ with $\beta_{i}=\mathrm{v} \cdot q_{i}+\mathrm{d} \cdot \alpha_{i}$ is strongly regular.

Proof The idea is that there are no constant names for $v$ and for $d$ in the signature of $\mathbb{H}$. As a consequence $\mathbb{H}$ is not a minimal datatype, i.e. it has proper substructures.

Let $a \in|\mathbb{H}|$, then for some meadow term $t\left(x_{1}, \ldots, x_{n}\right)$, not involving the constants $v$ and $\mathrm{d}, \mathbb{H} \models a=t\left(\beta_{1}, \ldots, \beta_{n}\right)$. Suppose that $\mathrm{v} \cdot a \neq 0$. Now $\mathrm{v} \cdot a=t\left(\mathrm{v} \cdot \beta_{1}, \ldots, \mathrm{v} \cdot \beta_{n}\right)=$ $\mathrm{v} \cdot t\left(q_{1}, \ldots, q_{n}\right)$.

It follows that $t\left(q_{1}, \ldots, q_{n}\right) \neq 0$ so that, because the $\beta_{i}$ are algebraically independent $t\left(\beta_{1}, \ldots, \beta_{n}\right) \neq 0$. Thus $a / a=\mathrm{v} \cdot t\left(q_{1}, \ldots, q_{n}\right) / t\left(q_{1}, \ldots, q_{n}\right)+\mathrm{d} \cdot t\left(\beta_{1}, \ldots, \beta_{n}\right) / t\left(\beta_{1}, \ldots\right.$, $\left.\beta_{n}\right)=\mathrm{v}+\mathrm{d}=1$.

One may define the structure $\mathbb{M}_{1}=\left(\mathbf{v} \cdot \mathbb{Q}_{0}^{d} \oplus \mathrm{d} \cdot \mathbb{R}_{0}^{d}, / /_{p}\right)$ to define an alternative division $/ f p$ as follows:

$$
\left(\mathrm{v} \cdot x+\mathrm{d} \cdot x^{\prime}\right) / f p\left(\mathrm{v} \cdot y+\mathrm{d} \cdot y^{\prime}\right)=\mathrm{v} \cdot(x / y)+\mathrm{d} \cdot x .
$$

The congruence induced by the structure $\mathbb{M}_{1}$ satisfies fracpair transparency (with $/ f p$ instead of $/$ ) for $x \neq 0$.

\subsection{True division with fracpair transparency}

We now consider the structure $\mathbb{M}_{2}=\left(\mathrm{v} \cdot \mathbb{Q}_{0}^{d} \oplus \mathrm{d} \cdot \mathbb{Q}_{0}^{d}, / f_{p}\right)$ with the division operation

$$
/_{f p}:\left(\mathrm{v} \cdot \mathbb{Q}_{0}^{d} \oplus \mathrm{d} \cdot \mathbb{Q}_{0}^{d}\right)^{2} \rightarrow \mathrm{v} \cdot \mathbb{Q}_{0}^{d} \oplus \mathrm{d} \cdot \mathbb{Q}_{0}^{d}
$$

defined by

$$
\left(\mathrm{v} \cdot x+\mathrm{d} \cdot x^{\prime}\right) / f_{p}\left(\mathrm{v} \cdot y+\mathrm{d} \cdot y^{\prime}\right)=\mathrm{v} \cdot(x / y)+\mathrm{d} \cdot((1-x / x) \cdot y+x \cdot(y / y)) .
$$

The structure $\mathbb{M}_{2}$ induces a congruence $\cong_{\mathbb{M}_{2}}$ on the set of terms over its signature. 
Table 7 Facts about true division $(-/ f p-)$ in the structure $\mathbb{M}_{2}$

$$
\begin{aligned}
& 2 / f p 3 \neq 4 / f p \\
& 0 / f p 3 \neq 0 / f p \\
& x / f p 0=0 \\
& 0 / f p 1 \neq 0 \\
& x \neq 0 \rightarrow x / f p 1=x \\
& \operatorname{val}(x / f p y+u / f p v)=\operatorname{val}(x / f p y)+\operatorname{val}(u / f p v) \\
& \operatorname{data}(x / f p y+u / f p v)=\operatorname{data}(x / f p y)+\operatorname{data}(u / f p v) \\
& x \neq 0 \vee u \neq 0 \rightarrow x / f p y+u / f p y=(x+u) /_{f p} y \\
& 0 / f p 1+0 / f p 1 \neq 0 / f p \\
& x / f p y=u /_{f p} v \rightarrow x / y=u / v
\end{aligned}
$$

Proposition 5.2.1 The congruence $\cong_{\mathbb{M}_{2}}$ is transparent for fracpairs.

Proof First notice that $x / f_{p} y=0$ if and only if $y=0$.

Consider the fracpairs $p \equiv \underline{k} / f p \underline{l}$ and $q \equiv \underline{m} / f p \underline{n}$ and assume $\mathbb{M}_{2} \vDash p=q$, then $p \neq 0 \neq q$ and $\operatorname{val}(p)=\operatorname{val}(q)$ and $\operatorname{data}(p)=\operatorname{data}(q)$. Thus $\mathbb{Q}_{0}^{d} \models \underline{k} / \underline{l}=\underline{m} / \underline{n}$ and $\mathbb{Q}_{0}^{d} \models(1-(\underline{k} / \underline{k})) \cdot \underline{l}+\underline{k} \cdot(\underline{l} / \underline{l})=(1-(\underline{m} / \underline{m})) \cdot \underline{n}+\underline{m} \cdot(\underline{n} / \underline{n})$, and because $l, n>0$ : $\mathbb{Q}_{0}^{d} \models(1-(\underline{k} / \underline{k})) \cdot \underline{l}+\underline{k}=(1-(\underline{m} / \underline{m})) \cdot \underline{n}+\underline{m}$. Now if $k=0$ then, with $\mathbb{Q}_{0}^{d} \models \underline{k} / \underline{l}=\underline{m} / \underline{n}$, also $m=0$ so that $\mathbb{Q}_{0}^{d} \models \underline{l}=\underline{n}$. In case $\bar{k} \neq 0$ also $m \neq 0$ and again $\mathbb{Q}_{0}^{d} \models \underline{l}=\underline{n}$. Now, with $\mathbb{Q}_{0}^{d} \models \underline{k} / \underline{l}=\underline{m} / \underline{n}$ also $\mathbb{Q}_{0}^{d} \models \underline{k}=\underline{m}$.

In order to assess flattening and value protection only expressions without occurrences of $-/-$ are to be taken into account.

Proposition 5.2.2 The mapping $-/ /_{p}-$ guarantees value protection.

Proof Suppose $\mathbb{M}_{2} \models p=q$ then $\mathbb{Q}_{0}^{d} \models \operatorname{val}(p)=\operatorname{val}(q)$. Here $\operatorname{val}(p)$ and $\operatorname{val}(q)$ are obtained by replacing $-/ f_{p}-$ by $-/-$ throughout these expressions. So the fracterms $p$ and $q$ have the same interpretation in $\mathbb{Q}_{0}^{d}$ under the assumption that $-/ f_{p}-$ stands for $-/-$ in that case.

Instead of flattening only a weaker assertion can be found:

Proposition 5.2.3 For closed there are $k, l \in \mathbb{Z}$, with $l>0$ and expressions $r$ and $s$ both possibly involving $-/-$ but not $-/ f_{p}-$ (i.e. ordinary meadow expressions) such that $\mathbb{M}_{2} \models t=(\mathrm{v} \cdot \underline{k}+\mathrm{d} \cdot r) / f p(\mathrm{v} \cdot \underline{l}+\mathrm{d} \cdot s)$.

Proof Write $t=\mathrm{v} \cdot \operatorname{val}(t)+\mathrm{d} \cdot \operatorname{data}(t)$. Flattening works within $\mathbb{Q}_{0}^{d}$ so given $t$ one finds integers $k$ and $l>0$ so that $\mathbb{Q}_{0}^{d} \models \operatorname{val}(t)=\underline{k} / \underline{l}$. Now consider $\left.p(r, s) \equiv(\mathrm{v} \cdot \underline{k}+\mathrm{d} \cdot r)\right|_{f p}(\mathrm{v}$. $\underline{l}+\mathrm{d} \cdot s)$. Straightforward calculation yields $p=\mathrm{v} \cdot \underline{k} / \underline{l}+\mathrm{d} \cdot((1-(r / r)) \cdot s+r \cdot(s / s))$. If $\operatorname{data}(t)=0$ choose $r=1, s=0$, otherwise choose $r=\operatorname{data}(t), s=1$, and in both cases $p(r, s)=\mathrm{v} \cdot \operatorname{val}(t)+\mathrm{d} \cdot \operatorname{data}(t)$.

Some facts concerning $\mathbb{M}_{2}$ are collected in Table 7.

What we conclude from the results of this section is that there is a meadow in which "fractions as values" can be understood in such a manner that fracpair transparency is guaranteed provided a new (different) definition of division is given, which makes use of division on the rationals (and original division in the meadow). 
Whether or not one considers the arithmetical datatype thus constructed to be sufficiently similar to ordinary arithmetic to call its elements numbers or "a kind of numbers" is a matter of taste. If so then one may hold that fractions may be considered a kind of numbers and may satisfy fracpair transparency at the same time.

\section{Concluding remarks}

Some terminology which is conventional in the area of fractions has not been introduced in Sect. 2. A more inclusive survey of such terminology is obtained with the following definitions:

- a fracpair $t / r$ with positive numerator $t$ is proper if $t<\cong r$,

- a fracpair with positive numerator that is not proper is said to be improper,

- a fracterm is composite if it is not flat.

- a unit fracterm (also unit fracpair) is a fracpair with 1 as its numerator,

- a term $\mathrm{t}$ is a mixed fracterm if it has the form $r+s$ or $-r-s$ with $r$ a whole term and $s$ a simple fracterm; for a mixed fracterm $r+s, r$ is called the integer part and $s$ is called the fractional part.

The latter terminology is somewhat unfortunate as a mixed fracterm is not a fracterm. In [2] it is shown that fractions involving a single variable can be brought in so-called mixed fraction format.

For the arithmetical datatypes $\widehat{\mathbb{Q}}_{0, g c d}^{d}$ and $\widehat{\mathbb{Q}}_{0, c f a r^{-}}^{d}$, the existence of a finite equational initial algebra specification remains an open question.

Acknowledgements We acknowledge the reviewers for very detailed critique and suggestions, including the proposal to use the phrase "compromised commutative ring" for the structures defined in Table 4.

Open Access This article is licensed under a Creative Commons Attribution 4.0 International License, which permits use, sharing, adaptation, distribution and reproduction in any medium or format, as long as you give appropriate credit to the original author(s) and the source, provide a link to the Creative Commons licence, and indicate if changes were made. The images or other third party material in this article are included in the article's Creative Commons licence, unless indicated otherwise in a credit line to the material. If material is not included in the article's Creative Commons licence and your intended use is not permitted by statutory regulation or exceeds the permitted use, you will need to obtain permission directly from the copyright holder. To view a copy of this licence, visit http://creativecommons.org/licenses/by/4.0/.

\section{References}

1. Bergstra, J.A., Bethke, I.: Note on paraconsistency and reasoning about fractions. J. Appl. Nonclass. Log. 25(2), 120-124 (2015). https://doi.org/10.1080/11663081.2015.1047232

2. Bergstra, J.A., Bethke, I., Hendriks, D.: Universality of univariate mixed fractions in divisive meadows. arXiv:1707.00499 [math.RA] (2017)

3. Bergstra, J.A., Bethke, I., Ponse, A.: Cancellation meadows: a generic basis theorem and some applications. Comput. J. 56(1), 3-14 (2013). https://doi.org/10.1093/comjnl/bxs028

4. Bergstra, J.A., Bethke, I., Ponse, A.: Equations for formally real meadows. J. Appl. Log. 13(2 part B), 1-23 (2015)

5. Bergstra, J.A., Hirshfeld, Y., Tucker, J.V.: Meadows and the equational specification of division. Theor. Comput. Sci. 410(12), 1261-1271 (2009)

6. Bergstra, J.A., Middelburg, C.A.: Inversive meadows and divisive meadows. J. Appl. Log. 9(3), 203-220 (2011)

7. Bergstra, J.A., Middelburg, C.A.: Division by zero in non-involutive meadows. J. Appl. Log. 13(1), 1-12 (2015) 
8. Bergstra, J.A., Middelburg, C.A.: Transformation of fractions into simple fractions in divisive meadows. J. Appl. Log. 16(1), 92-110 (2016)

9. Bergstra, J.A., Ponse, A.: Division by zero in common meadows. In: De Nicola, R., Hennicker, R. (eds), Software, Services, and Systems (Wirsing Festschrift), LNCS 8950, Springer, pp. 46-61 (Revised version) (2015). arXiv:1406.6878v3 [math.RA] (2019)

10. Bergstra, J.A., Ponse, A.: Fracpairs and fractions over a reduced commutative ring. Indag. Math. 27(3), 727-748 (2016). arXiv:1411.4410v2 [math.RA]

11. Bergstra, J.A., Tucker, J.V.: The rational numbers as an abstract data type. J. ACM 54(2), Article 7 (2007)

12. Bergstra, J.A., Tucker, J.V.: Division safe calculation in totalized fields. Theory Comput. Syst. 43, 410-424 (2008)

13. Bethke, I., Rodenburg, P.H.: The initial meadows. J. Symb. Log. 75(3), 888-895 (2010)

14. Bethke, I., Rodenburg, P.H., Sevenster, A.: The structure of finite meadows. J. Log. Algebraic Methods Program. 84(2), 276-282 (2015)

15. Carlström, J.: Wheels-on division by zero. Math. Struct. Comput. Sci. 14(1), 143-184 (2004)

16. Carlström, J.: Partiality and choice, foundational contributions. PhD. Thesis, Stockholm University. http:// www.diva-portal.org/smash/get/diva2:194366/FULLTEXT01.pdf (2005)

17. dos Reis, T.S., Anderson, J.A.D.W.: Construction of the transcomplex numbers from the complex numbers. In: Proceedings of WCECS 2014. http://www.iaeng.org/publication/WCECS2014/WCECS2014_pp97102.pdf. Aaccessed 23 Dec 2015 (2014)

18. Fandiño Pinilla, M.I.: Fractions: conceptual and didactic aspects. Acta Didact. Univ. Comen. Math. 7, 81-115 (2007)

19. Griesel, H.: Der quasikardinale Aspect in der Bruchrechnung. Der Mathematikunterricht 27(4), 87-95 (1981)

20. Kieren, T.E.: On the mathematical, cognitive, and instructional foundations of rational numbers. In: Lesh, R. (ed.), Numbers and Measurement: Papers from a Research Workshop, ERIC/SMEAC, Columbus OH, pp. 10-144 (1976)

21. Komori, Y.: Free algebras over all fields and pseudo-fields. Report 10, Faculty of Science, Shizuoka University, pp. 9-15 (1975)

22. Lortie-Forgues, H., Tian, J., Siegler, R.S.: Why is learning fraction and decimal arithmetic so difficult? Dev. Rev. 38, 201-221 (2015)

23. Ono, H.: Equational theories and universal theories of fields. J. Math. Soc. Jpn. 35(2), 289-306 (1983)

24. Padberg, F.: Didaktik der Bruchrechnung, Series: Mathematik, Primar-und Sekundarstufe (in German), 4th edn. Springer, Spektrum (2012)

25. Rollnik, S.: Das pragmatische Konzept für den Bruchrechenunterricht (in German.) PhD. thesis, University of Flensburg, Germany. https://www.zhb-flensburg.de/fileadmin/content/spezial-einrichtungen/zhb/ dokumente/dissertationen/rollnik/dissertation-flensburg-rollnik.pdf (2009)

26. Setzer, A.: Wheels (draft). http://www.cs.swan.ac.uk/ csetzer/articles/wheel.pdf (1997)

27. van Glabbeek, R.: The linear time—-branching time spectrum I. In: Bergstra, J.A., Ponse, A., Smolka, S.A. (eds.) Handbook of Process Algebra. Elsevier, Amsterdam (2001)

28. van Hiele, P.: Zouden we het rekenen met breuken misschien kunnen afschaffen (in Dutch). In: van Hiele, P., Begrip en inzicht, werkboek van de wiskundedidactiek, Muusses, Purmerend, Netherlands (1973)

Publisher's Note Springer Nature remains neutral with regard to jurisdictional claims in published maps and institutional affiliations. 